\title{
Vivencias de usuarias lesbianas sobre el proceso de atención perinatal en la región metropolitana, Chile, en 2016
}

Camila Fuenzalida Videla ${ }^{(a)}$

Andrea Velásquez Muñoz ${ }^{(b)}$

Videla CF, Muñoz AV. Experiences of lesbian users about the process of perinatal care in the metropolitan region of Chile in 2016. Interface (Botucatu). 2018; 22(66):777-87.

The study aimed to explore the experiences of perinatal care of lesbian users in the metropolitan area of Chile in 2016. It was a cross-sectional and phenomenological study with snowball sampling. In-depth interviews were carried out and the reports were interpreted using the content analysis technique. The interviews revealed positive experiences by lesbian users during their pregnancy, childbirth and postpartum periods, and an inclusive treatment, with no prejudice by healthcare providers. There is a broad acceptance by healthcare professionals of homosexual families and the visibility of homosexuality. The empowerment and self-esteem exhibited by the interviewed users favor the doctor-patient relationship. However, the alternatives to achieve motherhood are not available for the entire population because of their high cost.

Keywords: Perinatal care. Female homosexuality. Motherhood.
El objectivo de la investigación fue explorar las vivencias de la atención perinatal de usuarias lesbianas en la Región Metropolitana, Chile en 2016. Se trata de un estudio transversal exploratorio con enfoque fenomenológico, usando la estrategia muestral bola de nieve. Fueron realizadas entrevistas en profundidad con análisis de contenido. Se han experimentado vivencias favorables de las usuarias lesbianas sobre su proceso de gestación, parto y puerperio y un trato inclusivo y antidiscriminatorio de los proveedores de salud. Hay una aceptación generalizada de los profesionales de salud hacia las familias homoparentales y a la visibilización de la homosexualidad. El empoderamiento y la autoestima de las usuarias entrevistadas es un elemento que favorece la relación médico-paciente. Sin embargo, las alternativas para acceder a la maternidad no se encuentran asequibles para toda la población debido al alto costo que poseen.

Palabras clave: Atención perinatal. Homosexualidad femenina. Maternidad.

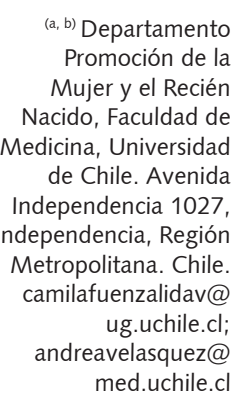

(a, b) Departamento Promoción de la Mujer y el Recién Nacido, Faculdad de de Chile. Avenida Independencia 1027, Independencia, Región Metropolitana. Chile. ug.uchile.cl; med.uchile.cl 


\section{Introducción}

La sexualidad se puede considerar como parte fundamental de la vida de las personas, la cual abarca diferentes aspectos como son el sexo, identidad de género, orientación sexual y también la maternidad.

Al hablar de familia se asocia comúnmente a la heteroparentalidad (madre, padre, hijos). Pero, existe la familia homoparental, entendiéndose en este estudio aquella compuesta por dos mujeres lesbianas o por una con hijo/a.

De acuerdo con lo anterior, existen diferentes posturas, España fue uno de los primeros países en aceptar los hogares homoparentales, además, junto con el Reino Unido y los países Escandinavos, permiten la adopción homoparental, en contraste con Francia, Italia o Alemania donde está prohibido. En un estudio realizado en Italia, sobre la percepción de mujeres lesbianas y bisexuales sobre la atención de los servicios médicos ha demostrado que la mayoría de lesbianas y mujeres bisexuales no revelan su orientación sexual al médico (internista, ginecólogo, entre otros) durante su atención. ¿No complica esto el encuentro entre las mujeres que tienen sexo con mujeres y los profesionales de la salud? La dificultad de las lesbianas y mujeres bisexuales para revelar su orientación sexual, por una parte, y la presuposición de los profesionales de la salud de la heterosexualidad de las pacientes, por la otra, crea obstáculos genuinos para la comunicación. Esta invisibilidad en la salud puede tener consecuencias importantes para el bienestar psicofísico de las consultantes. Si los profesionales de la salud subestiman sistemáticamente el número de estas pacientes, sus riesgos y problemas específicos de salud seguirán siendo inadvertidos; y las mujeres bisexuales y lesbianas pierden la oportunidad de comunicar sus problemas específicos y de estar cómodas durante la atención médica ${ }^{2}$. Además, se han podido identificar cuatro tipos de homofobias para con lesbianas: falta de reconocimiento de estas relaciones, asumir heterosexualidad, preguntas inapropiadas y negar los servicios directamente ${ }^{3}$, lo cual puede afectar de gran manera la relación proveedor de salud-usuaria lesbiana.

En América Latina y Central, Argentina aceptó legalmente la homoparentalidad (2010) y le siguió Colombia $(2011)^{4}$. Sin embargo, se detectaron 5 obstáculos principales por los que las lesbianas de Latinoamérica no ejercen plenamente su derecho a la salud: pobreza y deficientes sistemas locales de salud; prejuicio y lesbofobia entre el personal médico, especialmente ginecólogos y especialistas en salud mental; prejuicio y lesbofobia en el sistema legal y entre aquellos profesionales dedicados a combatir la violencia sexual y violencia doméstica; prejuicio e ignorancia en el interior de la misma comunidad lésbica; y otros problemas que afectan a las organizaciones lésbicas ${ }^{5}$.

Asimismo, es relevante reflexionar sobre la atención en salud que recibirán los padres y madres cuando manifiesten su deseo de paternidad/maternidad al proveedor de salud, porque, en ocasiones, éstos no reconocen la posibilidad de otra orientación sexual, lo que puede negar o invisibilizar la orientación de quién consulta, vulnerando sus derechos por omisión o negación ${ }^{6}$. En muchas ocasiones, según la Organización Mundial de la Salud (OMS), existe maltrato físico, verbal incluyendo la humillación a las mujeres, lo que atenta contras los derechos humanos ${ }^{7}$ e incluso contra los derechos sexuales y reproductivos dependiendo del lugar y el contexto de la atención.

En consecuencia, se observó en el estudio "An expression of love -midwives' experiences in the encounter with lesbian women and their partners" 6 realizado en Noruega, que los proveedores de salud, en específico las parteras, asimilan la atención de forma responsable ya que sienten que deben ser ellas las que abran los espacios de comunicación y deben adaptarse a las necesidades de las parejas lesbianas, siguiendo el instinto para poder realizar una atención adecuada en la que las consultantes no se sientan invadidas ni discriminadas. Aunque también consideraban difícil la atención, ya que no sabían cómo tratar a la co-madres debido a que no tenían claridad sobre su papel siendo incluso vista como una pareja masculina.

Por otra parte, se presentan diferentes aristas en la atención del proveedor de salud, desde la perspectiva de las mujeres atendidas, además depende del momento en que es brindada, es decir, si es previo a la gestación, en el desarrollo o posterior a esta. Cabe destacar que las investigaciones revisadas en el primer semestre del año $2016^{3,6,8-10}$ fueron en su mayoría de países extranjeros como el Reino Unido y los países Escandinavos, con muy poca información sobre América Latina y en especial, Chile. 
Los temas encontrados más a menudo fueron el prejuicio abierto y encubierto, es decir, aquel prejuicio que sentían las mujeres en la atención recibida, incluso demostrado a través de conversaciones sobre moralidad por parte del proveedor, y el encubierto por medio de prácticas que incomodaban a las mujeres, pero que no eran explícitas ${ }^{8}$. Si bien para ellas hay una potencial relación entre este prejuicio en la atención y su orientación sexual, a menudo existe un ejercicio de reinterpretación, donde se hace la asociación con la cultura organizacional y la personalidad de los profesionales de la salud, esta racionalización se usa como mecanismo protector, siendo una estrategia de las pacientes para mantener la dignidad e identidad personal de forma que adquieran el control de su protección ${ }^{8}$.

Otro tópico corresponde a la relevancia que se le otorga al conocimiento y confianza, considerando que ante la falta de conocimiento los proveedores de salud tienden a hacer una serie de preguntas irrelevantes e invasivas que pueden incomodar a las mujeres, teniendo que asumir ellas un rol educativo frente al profesional. Algunas disfrutan este rol al esperar que sea beneficioso tanto para ellas como para otras parejas en su situación. Por otra parte, pequeños gestos de apoyo que demuestren una actitud positiva, como estar de buen humor, respuestas relajadas y contacto visual, pueden resultar en sentimientos de apoyo, aceptación y empoderamiento, lo que genera una relación de confianza que es valorada por las mujeres ${ }^{8}$.

Un gran punto era la revelación de la orientación sexual al proveedor de salud, normalmente primero las madres lesbianas observaban la atención y dependiendo de cómo se sentían podían o no revelar su orientación, aunque en otras ocasiones la pareja homosexual llegaba a la consulta empoderada de su sexualidad y muy abierta ${ }^{10}$. Con relación al trato de la pareja en su conjunto, cabe enfatizar el trato que se daba a la co-madre ya que podía ser que no se incluyera, que se viera como el padre o en el caso del trabajo de parto, se veía de mejor manera ya que apoyaba mejor a la gestante comparada con una pareja masculina y encontraba un lugar en el espacio de la sala de forma más fácil, aún más si había sido madre anteriormente ${ }^{6}$.

Según los diferentes tópicos anteriormente nombrados con sus subtemas incluidos, se observa que una de las grandes preocupaciones que tienen las parejas homosexuales es el trato y aceptación que tendrán por parte de los proveedores de salud ya que muchas veces se sienten discriminadas por su orientación sexual e incluso pueden llegar a sentir temor por la atención que se les brindará pensando que le pueden causar algún daño a ellas o a sus recién nacidos/as, o que pueden ser juzgadas como malas madres. En países como Inglaterra las mujeres buscan matronas homosexuales, tener partos en casa o en hospitales que se definan como "amigables con lesbianas" para evitar exponerse a ese tipo de situaciones ${ }^{8}$.

Esto se hace necesario considerando que en Chile no existe un programa específico para la diversidad sexual, ni existen guías o protocolos de atención para mujeres homosexuales que serán madres, tampoco existe un rol claro dentro de la atención ni dentro de la sociedad para las parejas de ellas, las co-madres o segundas madres, las cuales son constantemente invisibilizadas. Por ejemplo, el Manual de Atención Personalizada en el Proceso Reproductivo inicia diciendo que: "El nacimiento es un momento determinante para la vida de todo ser humano y tanto el período previo como el inmediatamente posterior son decisivos para el desarrollo emocional, intelectual y social del niño, con una influencia central de la madre, el padre y la familia"11. (p. 8)

Sin considerar la posibilidad de que la familia a atender sea homoparental, por lo que se vuelve poco práctica para la atención de mujeres lesbianas y sus parejas en el contexto perinatal.

El estudio pretende enriquecer los espacios de discusión, poniendo énfasis en el proceso de atención perinatal vivenciado por lesbianas.

\section{Objetivo general}

Explorar las vivencias del proceso de atención perinatal de usuarias lesbianas en la Región Metropolitana, Chile en el año 2016. 


\section{Método}

La metodología fue de tipo cualitativa siendo un estudio transversal exploratorio con enfoque de tipo fenomenológico descriptivo expuesto por Edmund Husserl, el cual busca captar las experiencias individuales, el sentido global, el cómo las personas viven y dan sentido a la experiencia vivida y el significado de esa experiencia vivida en el cuerpo vivido. Para ello, se desplegaron una serie de acciones específicas planteadas por Martínez: se planteó y estructuró la entrevista en profundidad, en un primer momento, solo preguntas abiertas (pregunta de apertura) no relacionadas con el objetivo de la investigación y posteriormente el investigador se focalizó en aquellos aspectos que le resultaron más relevantes de la experiencia; se registró en una grabadora y analizó varias veces el todo de la entrevista entre tres investigadores y se acudió en dos ocasiones a los entrevistados, con el fin de aclarar ciertos significados que resultaban confusos para los investigadores. Una vez definidas las unidades de análisis, se fueron relacionando unas con otras, con el fin de comprender la vivencia en su totalidad ${ }^{12}$.

Las unidades de análisis correspondieron a usuarias lesbianas, madres, mayores de 18 años y que su atención perinatal haya ocurrido en el sistema público y/o privado de salud.

La estrategia muestral fue bola de nieve que: "consiste en pedir a los informantes que recomienden a posibles participantes. [...] permite acceder a personas difíciles de identificar"13. (p. 2)

Las organizaciones para el primer acercamiento fueron Agrupación Lésbica Rompiendo el Silencio, Familia Paralelas, Iguales y Línea Aborto Libre, en las cuales no es necesario tener autorización para contactar a sus participantes.

La técnica de recolección de datos fue entrevistas en profundidad. Primero existió un acercamiento entre el investigador y el entrevistado, donde se utilizó solo una pregunta de apertura, la cual permitió establecer un nexo. Posteriormente, aproximadamente treinta minutos después, se procedió a realizar preguntas abiertas sobre la experiencia de los entrevistados en relación al proceso perinatal, a sus vivencias sobre la maternidad, entre otras.

Se efectuó análisis de contenido. Fue utilizada una pauta con temas que indagaban en la atención perinatal. La cantidad de entrevistas dependió de la saturación de la información. El análisis de las entrevistas fue realizado por tres investigadores (una matrona, una psicóloga-matrona y una académica con experiencia en estudios cualitativos) con el fin de ampliar la perspectiva y profundidad del estudio y de enriquecer la producción de conocimiento. La codificación se realizó con el programa estadístico Atlas-Ti.

Se consideró la edad mayor de 18 años para evitar problemas legales y éticos posteriores. Por último, se resguardaron los nombres de las participantes. Todo lo anterior permitió favorecer el rigor y la calidad del estudio.

\section{Consideraciones éticas del estudio}

La investigación fue analizada a la luz de los postulados de la declaración de Helsinki, de la guía internacional de ética para la investigación biomédica que involucra sujetos humanos y de las guías de buena práctica clínica de ICH 1996. Sobre la base de la información entregada el estudio es aprobado por el Comité de Ética de la Facultad de Medicina de la Universidad (Proyecto n096-2016).

Para la realización de las entrevistas se solicitó que cada entrevistada firmara un consentimiento y que se recalcara el carácter voluntario de la participación.

Para resguardar la confidencialidad de las participantes se utilizó una codificación, la cual consistió en tomar las iniciales de cada nombre y cada apellido, junto con los cuatro últimos dígitos de la cedula de identidad y la edad.

\section{Limitaciones}

El acceso a la muestra podría ser entorpecido por la falta de visibilización de estas familias, lo cual fue resguardado mediante el contacto con organizaciones de diversidad sexual. 
Otra limitación está asociada al nivel socioeconómico, principalmente por el elevado costo que tiene el acceso a procedimientos de reproducción, por lo que se espera estratos sociales medios altos.

\section{Resultados}

Se realizaron oito entrevistas a usuarias lesbianas con edades entre los 25 y 39 años. El nivel socioeconómico era medio-alto. Las entrevistas duraron en promedio cincuenta minutos. Dos de ellas se atendieron en el sistema de salud público y privado y cuatro solo en el privado.

Los proveedores de salud con los que tuvieron contacto fueron ginecólogos, matronas, nutricionistas, enfermeras y técnicos de enfermería.

El material transcrito fue analizado para definir las categorías acordes con los objetivos planteados.

\section{A) Sistema de Salud}

Dentro del sistema de salud chileno, existen dos categorías: privado y público. El sistema de salud público se considera como el Fondo Nacional de Salud (FONASA) y es el que cubre a la mayor parte de la población; en cambio el sistema privado está constituido por las Instituciones de Salud Previsional (ISAPRES) que cubren un porcentaje menor de población por el alto costo que posee encontrarse en este sistema ${ }^{14}$.

Se utilizaron ambos sistemas de salud disponibles. Hay diferencias en el mismo sistema de salud (público) ya que una usuaria no tenía problemas para ir al consultorio, en cambio la otra abandonó la atención primaria pública por la mala calidad de atención.

... acá al consultorio de $\mathrm{X}$ comuna, me dieron hora con la nutricionista, con la matrona, con todo. [...] en $X$ comuna no se llena nada, te dan la hora a las 11 y a las 11 te atienden..." (SPM246438)

...yo llegaba un día y decían no es que cambiaron la hora y no avisaban, [...] Varias veces pasó eso, fue un conjunto de cosas en el consultorio que fue malo. (CPE620525)

Las usuarias relacionaban la calidad de la atención con el tiempo y ambiente en el que se desempeñaban los proveedores según el sistema de salud.

Creo que tiene más que ver porque en el sector privado están tranquilos trabajando, se dan el tiempo para atenderte. [...] (APE122052)

Finalmente, existe cierta seguridad con el sistema de salud público por los servicios que posee, los cuales muchas veces no están disponibles en el sistema privado, como lo son ciertas urgencias maternas y neonatales, lo que puede cambiar la decisión de la usuaria sobre la atención del parto.

[...] en el hospital, si hay alguna urgencia se tiene altiro una urgencia para la guagua, una para la mamá, no así pasa en algunas clínicas, que si pasa algo se tiene que partir al hospital de emergencia... ese igual fue un factor que influyó [...] (SPM246438)

\section{B) Motivaciones para ser madre}

Las entrevistadas afirmaron que su deseo por la maternidad era parte de su proyecto de vida. Era importante compartir este deseo ya que nos revelan que, al comenzar su relación, fue un tema de conversación y que, en alguna etapa, sintieron la necesidad de hacerlo realidad. Además, destaca que el deseo de extender la familia no es diferente al de familias heteroparentales. 
[...] una de las preguntas era si queríamos tener hijos y las dos dijimos que sí: "tu querís tener hijos", "sí" ... Entonces las dos siempre tuvimos esa idea... (SPM246438)

[...] la motivación es la de todas las parejas, [...] que la familia no se quede en dos no más, no es muy distinta a la motivación de una familia heterosexual. (CSA744634)

\section{C) Acceso a la maternidad}

Existen diferentes formas para acceder a la maternidad. Dentro de estas, se encuentran el contacto con hombres homosexuales, amigos o a través de clínicas de reproducción. Lo cual, propicia diferencias en los riegos (infección VIH) y el costo.

...hicimos un blog explicando que éramos una pareja, que queríamos un hombre gay [...] resulta que después cuando se fue a Australia nos dice "chiquillas, saben que tengo el VIH" y nosotras casi nos morimos... (SPM246438)

....para hacer esto hay que tener lucas, si no tení plata, lo veo poco probable [...], todas las clínicas de reproducción son caras. (COJ414537)

Se encuentran diferentes percepciones sobre el costo de una inseminación artificial y la posible necesidad de una política pública. Se puede ver desde que la inseminación no es cara o que es muy cara ya que no hay código FONASA para disminuir el costo.

... la inseminación no es cara, es un tratamiento [...] lo que es caro es importar las cuatro muestras, [...] si tú no tienes problemas de fertilidad, te sale 200 lucas [...] (CBB207335)

[...] para qué decirte el costo del tratamiento de fertilidad que, además, no están cubiertos por ningún sistema de salud porque no están codificados por Fonasa. (CSA744634)

\section{D) Revelación de la orientación sexual a los proveedores de salud}

La revelación de la orientación sexual ocurre en la primera consulta y de forma espontánea.

Existieron reacciones de desconcierto ante la revelación, pero no fue percibida como discriminación sino más bien, atribuida a la edad del proveedor y al desconocimiento del tema.

En otras experiencias, la información no causó discriminación, se aceptó e hizo partícipe a la comadre en el proceso, lo que nos muestra aceptación hacia estas familias.

... con el Doctor fue como chocante cuando le dijimos "es que ambas somos las mamás del bebé, somos homosexuales", pero al rato miró como "ah! Ahora entiendo" era viejito, de edad, [...], pero no nos sentimos mal ni nada [...] (CPE620525)

A todos les dijimos altiro y en realidad, no hubo ningún problema, no nos sentimos discriminadas [...], ella tenía que entrar a la neo y para que le dieran información la pusieron como "la otra mamá. (MOS143739)

\section{E) Experiencias pre y posnatales}

Dentro de las experiencias prenatales se encuentran las expectativas e importancia del lugar dónde se desarrolló el trabajo de parto.

Se destaca dentro de la experiencia postnatal el cambio de habitación en la clínica y considerar a la "otra mamá" como parte de la familia incluyéndola en la epicrisis. 
...fue un trabajo de parto rico, en una sala integral, con música, luces bajas, masajes...Mucho cariño [...] (CBB207335)

....allá incluso nos cambiamos de pieza porque los compañeros de pieza eran evangélicos [...] fue algo importante que nos cambiaran. (CPE620525)

Incluso en la epicrisis del alta dice: mamá "yo", papá: sale la Viví así ningún atado [...] (COJ414537)

\section{F) Percepciones}

Las percepciones de la atención fueron positivas tanto en el sistema de salud público como privado e incluso recomiendan sus experiencias.

Se destaca que perciben algunas acciones como realizadas por voluntad del personal y no por normas o protocolos establecidos.

en la clínica " $X$ " siempre nos hicieron sentir bien [... y yí, recomiendo mi experiencia.

(MOS143739)

...la clínica donde lo tuve fue demasiado cariñoso todo, muy buena onda, te tratan súper bien

[...] (CBB207335)

[...] todo esto que te cuento yo es porque tuvimos suerte no más, es una cuestión de voluntades,

[...] o sea si ellos querían, tenían todas las facultades para dejarla afuera. (CSA744634)

\section{G) Relación con el proveedor de salud}

La importancia en la relación con el proveedor de salud se asocia con ciertos aspectos como la confianza que le transmita e incluso el proporcionar el número de whatsapp. Además, de la inclusión de la pareja a los controles. Por otra parte, la relación puede trascender la atención cerrada, creando lazos en los cuales, se considera importante seguir en contacto y visitar a las personas de las que recibieron un trato respetuoso y cariñoso. Lamentablemente, en un caso la matrona del consultorio presionaba a la usuaria para que le dijera el nombre del progenitor aun sabiendo la orientación sexual de ella, lo que claramente atenta contra los derechos del paciente y daña la relación con el proveedor y el sistema de salud.

[...] ahí empezamos a ir con la Viví, [...], el Doctor la quería caleta, siempre la invitaba a pasar [...] (COJ414537)

Las matronas nos dicen "las bombitas" porque estábamos a punto de explotar [...]. De hecho, las pasaremos a ver este mes cuando vayamos al control. (MOS143739)

Con la matrona sí me sentí súper mal, me decía "pero dame el nombre del papá" y yo le decía: pero si no hay papá y me decía: iah! ¿Te metiste con cualquiera? [...] (CPE620525)

\section{Discusión}

Las experiencias han sido, en general, favorables. Las usuarias no perciben discriminación y la búsqueda del hijo/a no se ha visto imposibilitada por su orientación sexual aun considerando el alto costo de las clínicas de reproducción y el potencial riesgo al realizar una inseminación artificial "artesanal". 
Dentro de las atenciones según sistema, se pueden observar diferencias que existen entre profesionales de la misma profesión, con el cuestionamiento correspondiente sobre qué motiva que la atención cambie entre centros de salud (público versus privado) del mismo sistema.

Fue utilizado mayormente el sistema de salud privado, lo que posiblemente se deba al nivel socioeconómico al que pertenecían las entrevistadas, así como también, a las proyecciones que tenían de la gestación como tener el parto en una clínica o pensionado y no en una sala común de hospital, por los prejuicios que tenían sobre la atención pública.

Un punto importante que discutir sobre el período preconcepcional, es la forma en que logran la gestación. Cabe destacar que siete de las entrevistadas asistieron a una clínica de reproducción, donde el tema fue el costo de las muestras ya que es necesaria cierta cantidad de dinero para importarlas debido a que en Chile no existe banco de esperma, lo que restringe a usuarias de menor nivel socioeconómico.

Esta situación vulnera los derechos sexuales y reproductivos ya que no permite que las usuarias decidan cuándo tener hijos/as y el espaciamiento entre ellos $^{15}$. Si una usuaria no tiene el dinero necesario para importar las muestras y realizar el procedimiento, la única posibilidad que tiene es hacer partícipe a un hombre para realizar una inseminación artificial artesanal o coito, exponiéndose a los riesgos que esto conlleva tanto biológica (Infecciones de transmisión sexual) como legalmente ya que, además, él tendría más derecho sobre el recién nacido que la madre no biológica si quisiera reconocerlo. Esto demuestra la urgencia de una ley de filiación para parejas homosexuales o matrimonio igualitario.

Según esto último, Chile se encuentra atrasado ya que tanto en Argentina como Colombia se acepta la homoparentalidad y existe matrimonio igualitario. Frente a esto, surge la interrogante ¿Qué hace falta para que en Chile se apruebe? En la presente investigación se puede ver que las experiencias y percepciones de las usuarias revelan que los proveedores de salud están abiertos a asistir y atender a las usuarias lesbianas sin discriminación. Ello constituye una señal favorable para los tiempos actuales en los que la diversidad sexual está teniendo mayor activismo, lo que contrasta con lo expuesto en la introducción ya que no se encuentra ningún prejuicio abierto ni encubierto que pudiera afectar la vivencia de la usuaria, siendo beneficioso para que éstas confíen en el sistema de salud y la atención brindada.

A pesar de los resultados optimistas arrojados en el estudio, hay que considerar si esta percepción globalmente positiva pueda tener relación con el nivel de educación, con las normas sociales impuestas o se deba al miedo a la sanción jurídica. A partir del año 2012 en Chile se promulgó la ley 20.609, que establece medidas contra la discriminación, más conocida como Ley antidiscriminación, o por su nombre no oficial Ley Zamudio, es una ley chilena que tiene por objetivo fundamental instaurar un mecanismo judicial que permita restablecer eficazmente el imperio del derecho toda vez que se cometa un acto de discriminación arbitraria, estableciendo un procedimiento judicial y medidas sancionatorias en caso de comisión de un acto de ese tipo ${ }^{16}$. A partir del año 2012, varias organizaciones influyentes en el tema de diversidad sexual han utilizado este control social frente a episodios de discriminación. Al parecer en Chile, es necesario el control social y sanciones penales para lograr un entorno incluyente y no discriminatorio o quizás solo es un elemento por considerar.

También existe un elemento que podría estar contribuyendo en la visibilización de la homosexualidad, es el sistema de salud privado, porque se tiene en el inconsciente colectivo que, al pagar por un servicio, se poseen más derechos, lo cual de una u otra manera podría empoderar a las usuarias en relación con sus derechos sexuales y reproductivos. Sumado a lo anterior existe mayor aceptación de la diversidad sexual en los ambientes sociales más favorecidos ${ }^{17}$.

En esta investigación, a diferencia de la expuesta en la introducción, destaca que la revelación de la orientación sexual, existen diferencias porque en la publicación se esperaba primero crear un lazo de confianza $^{10}$, en contraste con esta investigación donde se realizó en la primera consulta y no se recibió discriminación ni malos tratos. Quizás lo anterior, se podría explicar en que la mayoría de las usuarias 
provenían de un nivel socioeconómico medio-alto y poseían una autoestima positiva y una gran confianza, ya que revelaban su homosexualidad y sus necesidades específicas en la primera cita con el profesional de salud, situación que favorece el acceso y la comunicación fluida con los profesionales de salud.

Hay una aceptación generalizada hacia las familias homoparentales incluyendo a la co-madre -si está presente- en el proceso. Este último punto, se encuentra en concordancia con la bibliografía expuesta ${ }^{10}$, de hecho, la consideran con los mismos derechos para los padres heterosexuales como lo es entregar información sobre el estado de un recién nacido hospitalizado pudiendo no habérsele considerado por no existir una filiación sanguínea o legal; esto demuestra la comprensión y aceptación del equipo interdisciplinario con la familia homoparental desde una perspectiva biopsicosocial con la importancia requerida en este proceso vital.

Cabe destacar, que a pesar de la percepción positiva que tienen las usuarias de los profesionales de salud, existe cierta justificación o normalización de expresiones de homofobia de los profesionales de salud. De cierta manera las usuarias lesbianas tratan de negar ciertos comentarios de los proveedores de salud y racionalizarlos, ya sea alusivos a la edad o a la personalidad.

En este estudio, si bien existía la limitación del nivel socioeconómico, no se incorporó en los criterios de inclusión y/o exclusión el sistema de salud utilizado. De todas maneras, solo existieron dos entrevistadas que utilizaron de alguna forma el sistema público de salud. Esto da una visión enfocada en el por qué ocurre y qué se podría hacer para cambiarlo. Como se ha dicho anteriormente, el alto costo de las clínicas de reproducción fue un factor influyente en el estatus socioeconómico y que seguirá existiendo mientras no se realicen las políticas públicas necesarias para brindar la oportunidad a más personas siendo o no homosexuales, de acceder a una alternativa de reproducción sin gran repercusión monetaria, lo que también conllevaría el respetar los acuerdos firmados en las convenciones de derechos humanos y sexuales y reproductivos. En consecuencia, no parece ético de parte del sistema de salud público, no poseer alternativas para usuarias, que, en estos momentos, no pueden acceder a las clínicas de reproducción por el costo que posee y no tenerlo considerado dentro de sus prestaciones de salud.

Por otra parte, si se analizan los derechos sexuales, a los cuales Chile adscribe, se encuentra que se vulneran varios de estos, tales como el derecho a la igualdad y a la no-discriminación ya que las familias homoparentales no poseen los mismos derechos que las heteroparentales tanto legales como en las alternativas de tener hijos/as. Además, se vulneran los derechos siete y ocho que tienen relación con tener el grado máximo alcanzable de salud y de gozar de los avances científicos y de los beneficios que de estos resulten porque si bien pueden acceder a la maternidad a través de la inseminación artificial, se debe contar con el ingreso económico suficiente para poder costear el proceso y los controles posteriores, lo que incluye una diferencia entre los diferentes niveles socioeconómicos e incluso puede verse alguna relación entre el poder adquisitivo, por ende la clase social, y las opciones disponibles. Y por último, se vulnera totalmente el derecho número 12 que corresponde a: "El derecho a decidir tener hijos, el número y espaciamiento de los mismos, y a tener acceso a la información y medios para lograrlo"15 (p. 3). Los derechos sexuales se encuentran dentro de los derechos humanos, a los cuales Chile también adscribe, por lo que, al vulnerar los derechos sexuales de las usuarias lesbianas, también está vulnerando sus derechos humanos ${ }^{18}$. Lo que demuestra que existe una contradicción entre lo que el estado de Chile debe proteger y lo que se realiza dentro del país.

Finalmente, hay que tener en consideración que las familias homoparentales irán en aumento porque la diversidad sexual ha comenzado a visibilizarse en la sociedad. Por lo tanto, debería ir acorde con los avances en los ámbitos legales, sanitarios y educacionales, tanto para los proveedores de salud y la sociedad en general, ya que es la única manera de evitar los prejuicios y discriminación. Hace falta concienciar dentro del sistema sanitario el respeto a las necesidades de las usuarias homosexuales y desarrollar las destrezas que permitan darles respuesta. 


\section{Colaboradores}

Ambas autoras han participado de forma activa en la investigación para la realización de este artículo, considerando la búsqueda de referencias bibliográficas, discusiones sobre los artículos encontrados, su validez según la antigüedad. Además, ambas participaron en el contacto de entrevistadas, realización de entrevistas y análisis de contenido de éstas para obtener los resultados y realizar, en conjunto, la discusión posterior. Finalmente, se realizó la revisión final del artículo por parte de ambas autoras, de forma paralela, para realizar posteriormente, la última discusión previo a la entrega del artículo.

\section{Referencias}

1. Co-padres. Homoparentalidad: desigualdad por el mundo [Internet]. 2011 [citado 6 May 2015]; 1. Disponible en: http://chicachica.com/BIBLIOTECA_files/copadres $2 \% 20$ homoparentalidad-1.pdf

2. Asociación Internacional de Lesbianas-Gays-Bisexuales-Trans e Intersex. La salud de las lesbianas y mujeres bisexuales: cuestiones locales, preocupaciones comunes [Internet]. Bruselas; 2007 [citado 15 Jun 2016]; 2-67. Diponible en: http://www.felgtb.org/rs/525/ d112d6ad-54ec-438b-93584483f9e98868/196/filename/2

3. Hayman B, Wilkes L, Halcomb EJ, Jackson D. Marginalised mothers: lesbian women negotiating heteronormative healthcare services. Contemp Nurse [Internet]. 2013 [citado 5 May 2015]; 44(1):120-7. Disponible en: https://www.ncbi.nlm.nih.gov/ pubmed/23721394

4. Perrotta G. Concepciones de sujeto/paciente en salud sexual y reproductiva: acceso de mujeres lesbianas y bisexuales [Internet]. En: Anales del $6^{\circ}$ Congreso Internacional de Investigación y Práctica Profesional en Psicología, 21a Jornadas de Investigación Décimo Encuentro de Investigadores en Psicología del MERCOSUR; 2014; Buenos Aires. Buenos Aires: Acta Académica; 2014. p. 49-52 [citado 6 May 2015]. Disponible en: https://www. aacademica.org/000-035/249.pdf

5. Eiven L, Sardá A, Villalba V. Lesbianas, salud y derechos humanos desde una perspectiva latinoamericana. Rev Mujer Salud. 2007; 2:43-54.

6. Spidsberg BD, Sørlie V. An expression of love - midwives' experiences in the encounter with lesbian women and their partners. J Adv Nurs [Internet]. 2012 [citado 10 May 2015]; 68(4):796-805. Disponible en: https://www.ncbi.nlm.nih.gov/pubmed/21781147

7. Organización Mundial de la Salud. Prevención y erradicación de la falta de respeto el maltrato durante la atención del parto en centros de salud. Declaración de la OMS Internet]. 2014 [citado 10 May 2015]; 1-4. Disponible en: //apps.who.int/iris/ bitstream/10665/134590/1/WHO_RHR_14.23_spa.pdf?ua=1

8. Dahl B, Margrethe Fylkesnes A, Sørlie V, Malterud K. Lesbian women's experiences with healthcare providers in the birthing context: a meta-ethnography. Midwifery [Internet]. 2013 [citado 10 May 2015]; 29(6):674-81. Disponible en: https://www.ncbi.nlm.nih.gov/ pubmed/22901600

9. Ruppel EH, Karpman HE, Delk CE, Merryman M. Online maternity information seeking among lesbian, bisexual, and queer women. Midwifery [Internet]. 2017 [citado $20 \mathrm{Abr}$ 2017]; 48:18-23. Disponible en: https://www.ncbi.nlm.nih.gov/pubmed/28314179

10. Lee E, Taylor J, Raitt F. "It"s not me, it's them': how lesbian women make sense of negative experiences of maternity care: a hermeneutic study. J Adv Nurs [Internet]. 2011 [citado 10 May 2015]; 67(5):982-90. Disponible en: https://www.ncbi.nlm.nih.gov/ pubmed/21214618 
11. Ministerio de Salud (AR). Manual de atención personalizada en el proceso reproductivo [Internet]. Buenos Aires: Departamento Ciclo Vital, División Prevención y Control de Enfermedades, Subsecretaría de Salud Pública, Ministerio de Salud; 2008 [citado 8 May 2015]; 6-356. Disponible en: http://web.minsal.cl/portal/url/ item/795c688fd24acd68e04001011e013bfb.pdf

12. Martínez M. El comportamiento humano. 2a ed. México: Trillas; 2002.

13. Martín-Crespo MC, Salamanca AB. El muestreo en la investigación cualitativa. Nure Investigación [Internet]. 2007 [citado 20 Abr 2017]; 27(1):1-4. Disponible en: http:// www.nureinvestigacion.es/OJS/index.php/nure/article/view/340/330

14. Becerril-Montekio V, de Dios Reyes J, Manuel A. Sistema de salud de Chile. Salud Pública Mex. [Internet]. 2011 [citado 20 Abr 2017]; 53 Supl 2:132-42. Disponible en: http://www.scielo.org.mx/scielo.php?script=sci arttext\&pid =S0036-36342011000800009

15. World Association for Sexual Health. Declaracion de los Derechos Sexuales [Internet]. 2014 [citado 10 May 2015]. Disponible en: http://www.worldsexology.org/wp-content/ uploads/2013/08/declaracion_derechos_sexuales_sep03_2014.pdf

16. Ley $n^{\circ} 20.609$, de 12 de julio de 2012. Ley antidiscriminación. Diario Oficial de la República de Chile. 24 Jul 2012.

17. Instituto Nacional de la Juventud. Jóvenes sexual de hoy y diversidad percepciones [Internet]. 2014 [citado 10 May 2015]. Disponible en: http://www.injuv.gob.cl/portal/ wp-content/files_mf/revista10_final.pdf

18. Onufer S, Muntarbhorn V. Principios de Yogyakarta [Internet]. 2007 [citado 5 May 2016]; 5-38. Disponible en: http://www.refworld.org/cgi-bin/texis/vtx/rwmain/ opendocpdf.pdf?reldoc $=\mathrm{y} \&$ docid $=48244 \mathrm{e} 9 \mathrm{f} 2$

Videla CF, Muñoz AV. Vivências de usuárias lésbicas sobre o processo de atenção perinatal na região metropolitana do Chile em 2016. Interface (Botucatu). 2018; 22(66):777-87.

O objetivo do trabalho foi percorrer as vivências da atenção perinatal, de usuárias lésbicas na região metropolitana do Chile em 2016. Trata-se de estudo transversal de exploração com abordagem fenomenológica, utilizando a amostragem em bola de neve. Foram realizadas entrevistas em profundidade, com análise de conteúdo. Foram observadas vivências favoráveis das usuárias lésbicas acerca de seus processos de gestação, parto e puerpério, e um tratamento inclusivo e sem discriminação dos prestadores de saúde. Existe uma geral aceitação dos profissionais da saúde em relação às famílias homossexuais e à visibilidade da homossexualidade. O empoderamento e a autoestima das usuárias entrevistadas é um elemento que favorece o relacionamento médico-paciente. Porém, as alternativas para alcançar a maternidade não são acessíveis para toda a população, devido ao alto custo que elas têm.

Palavras-chave: Atenção perinatal. Homossexualidade feminina. Maternidade.

Sometido en 05/05/17. Aprobado en 29/06/17. 
